\title{
Estructura y dinámica de aplicación de los modelos de evaluación institucional para la calidad educativa
}

\author{
Emma Melida Bermeo Paredes. \\ emmamelida@hotmail.com \\ Universidad del Zulia \\ Mineira Finol de Franco \\ mineirafinoldefranco@gmail.com \\ Universidad del Zulia
}

\section{Resumen}

La presente investigación tuvo como objetivo describir la estructura y dinámica de aplicación de los modelos de evaluación institucional para la calidad educativa. La orientación metodológica planteó como estrategia la revisión documental entre los cuales se analizaron textos, leyes, reglamentos e informes, relacionados con la evaluación institucional. Se concluye que el modelo de Malcolm Baldrige tiene como estructura los criterios Liderazgo, Planificación, Orientación al cliente, Información y análisis, Orientación a las personas, Gestión de los procesos y Resultados; el modelo EFQM presenta nueve criterios que se agrupan en dos bloques que son agentes facilitadores y resultados; en el caso del modelo CINDA plantea el análisis y reflexión sistemática acerca del desarrollo de la educación superior y de sus implicaciones en diversos ámbitos, estableciendo la autoevaluación como proceso interno permanente; el Modelo de Gento Palacios se basa en indicadores y predictores. En el caso del CONEA plantea tres etapas basados en la autoevaluación, la evaluación externa y la acreditación, y finalmente el CEAACES se basa en planificar, coordinar y ejecutar las actividades del proceso de evaluación, acreditación, clasificación académica y aseguramiento de la calidad de la educación superior.

\section{Palabras clave}

Estructura de evaluación institucional, Dinámica de aplicación, Calidad educativa, Evaluación institucional 


\title{
Structure and dynamics of application of institutional evaluation models for educational quality
}

\begin{abstract}
The purpose of this research was to describe the structure and dynamics of application of the institutional evaluation models for educational quality. The methodological orientation proposed as a strategy from a documentary review among texts, laws, regulations and reports, related to the institutional evaluation were analyzed. It is concluded that the Malcolm Baldrige model has as its structure in the criteria Leadership, Planning, Customer orientation, Information and analysis, People orientation, Process management and Results; The EFQM model presents nine criteria that are grouped into two blocks that are facilitating agents and results; In the case of the CINDA model, it raises the systematic analysis and reflection on the development of higher education and its implications in various fields, establishing self-assessment as a permanent internal process; The Gento Palacios Model is based on indicators and predictors. In the case of CONEA, it proposes three stages based on self-evaluation, external evaluation and accreditation, and finally CEAACES is based on planning, coordinating and executing the activities of the evaluation, accreditation, academic classification and quality assurance process for higher education.
\end{abstract}

\section{Keywords}

Institutional evaluation structure, Application dynamics, Educational quality, Institutional evaluation 


\title{
Estrutura e dinâmica de aplicação de modelos de avaliação institucional para qualidade educacional
}

\begin{abstract}
Resumo
O objetivo desta pesquisa foi descrever a estrutura e dinâmica de aplicação dos modelos de avaliação institucional para a qualidade educacional. A orientação metodológica propôs como estratégia a revisão documental entre os quais foram analisados textos, leis, regulamentos e relatórios relacionados à avaliação institucional. Conclui-se que o modelo de Malcolm Baldrige tem como estrutura os critérios Liderança, Planejamento, Orientação para o Cliente, Informação e análise, Orientação para Pessoas, Gerenciamento de Processos e Resultados; O modelo EFQM apresenta nove critérios agrupados em dois blocos que facilitam agentes e resultados; No caso do modelo CINDA, levanta a análise e reflexão sistemáticas sobre o desenvolvimento do ensino superior e suas implicações em vários campos, estabelecendo a auto avaliação como um processo interno permanente; O Modelo Gento Palacios é baseado em indicadores e predito rês. No caso do CONEA, propõe três etapas com base na auto avaliação, avaliação externa e credenciamento e, por fim, o CEAACES baseia-se no planejamento, coordenação e execução das atividades do processo de avaliação, credenciamento, classificação acadêmica e garantia da qualidade. Educação superior.
\end{abstract}

\section{Palavras chave}

Estrutura de avaliação institucional, Dinâmica de aplicação, Qualidade educacional, Avaliação institucional. 


\section{Introducción}

En el mundo de hoy en pleno siglo XXI es importante considerar el trabajo que se realiza en las instituciones educativas y para ello es relevante destacar los modelos y órganos que permiten realizar la evaluación sobre la calidad, en función de esto en la presente investigación se describe la estructura y dinámica de los modelos de Malcolm Baldrige, EFQM, CINDA y Gento Palacios así como las modelos asumidos por organizaciones CONEA, CEAACES (Ecuador).

En este estudio se muestra algunos modelos que se utilizan para evaluar la calidad educativa en las universidades como espacio de formación de recursos humanos, en el caso de Ecuador donde la educación ha sido evaluada por la Secretaría Nacional de Educación Superior, Ciencia, Tecnología e Innovación (SENESCYT) la cual es la institución rectora de la política pública en el campo de la Educación Superior, la Ciencia, la Tecnología, la Innovación y Saberes Ancestrales mediante la coordinación y articulación de sus acciones entre el sector público y los sectores productivos públicos y privados, cuyos resultados son la semilla para el desarrollo del país.

En este orden, se muestran diversas fuentes bibliográficas que profundizan en los modelos de evaluación institucional para la búsqueda de la calidad educativa, argumentando sus postulados teóricos, estructura y dinámica de aplicación con base a las características particulares de las organizaciones de educación superior los cuales fueron sistematizados en este estudio, lo cual servirá de fundamento para otras investigaciones relacionada con la temática. Es así, que considerando lo expuesto por Tünnermann (2002) citado por Finol de Franco y Col (2002) quien señala que la calidad educativa toma una importancia sin precedentes dentro de los procesos de globalización y competitividad, al punto que es prioridad su inserción en las agendas educativas de todos los países.

En este sentido Muñoz explica que:

la educación es de calidad cuando está dirigida a satisfacer las aspiraciones del conjunto de los sectores integrantes de la sociedad a la que está dirigida; si, al hacerlo, se alcanzan efectivamente las metas que en cada caso se persiguen; si es generada mediante procesos culturalmente pertinentes, aprovechando óptimamente los recursos necesarios para impartirla y asegurando que las oportunidades de recibirla y los beneficios sociales y económicos derivados de la misma se distribuyan 
en forma equitativa entre los diversos sectores integrantes de la sociedad a la que está dirigida (2003, p.51).

Por otra parte, Graells (2002) afirma que la calidad en la educación asegura a todos los jóvenes la adquisición de los conocimientos, capacidades destrezas y actitudes necesarias para equipararles para la vida adulta.

Blotta, Corengia y Carranza (2003) investigaron el tema de la identidad institucional como uno de los indicadores que determinan la calidad en el nivel universitario, siguiendo los criterios utilizados por la Red Universitaria de Evaluación de la Calidad. En su estudio de investigación, realizan la distinción entre calidad declarada y calidad percibida, donde el primer término hace referencia a lo declarado en los documentos institucionales (ideario, misión, valores, cultura) y el segundo término, a la calidad percibida por la comunidad de profesores, alumnos, graduados y no docentes. Agregan además el concepto de calidad sugerida, bajo el cual agrupan las sugerencias de mejora realizadas por los diferentes actores de la comunidad universitaria.

Para Senlle y Gutiérrez la calidad es

Un espíritu de vida, un cambio de clima, el deseo de hacer las cosas bien obtener resultados y mejorar puntos. Por otra parte, la calidad la hacen las personas tanto directivos, técnicos, administrativos, como docentes, quienes conociendo el uso y aplicación de técnicas actuales de calidad para que puedan aplicarlas mejorando permanentemente su gestión. Incluso los alumnos y sus padres son actores de un sistema de calidad (2005:3).

En este sentido destaca la importancia de generar un servicio educativo de calidad donde se satisfaga las expectativas y necesidades de los estudiantes, de la sociedad en general e igualmente de quienes desarrollan funciones y tareas en las instituciones, cada uno en referencia a la misión, visión, objetivos definida por la organización. En el caso de Ecuador desde lo expuesto por la Secretaría Nacional de Educación Superior, Ciencia, Tecnología e Innovación (SENESCYT, 2017) en el nivel de educación superior se ha planteado la publicación de la Ley Orgánica de Educación Superior (LOES) en el 2010, donde se expone que el Ecuador inició un proceso orientado a garantizar el derecho a la Educación Superior de calidad, que propenda a la excelencia, al acceso universal, a la permanencia y egreso sin discriminación lo cual es socialmente justificable. De igual forma durante los últimos años, el acceso a la Educación Superior se ha incrementado en 
varios puntos porcentuales, lo que muestra un enorme esfuerzo por ampliar la cobertura. $\mathrm{El}$ origen de este cambio radica en acciones progresivas que permiten ir eliminando las barreras de acceso.

Los sectores más pobres del Ecuador han duplicado su acceso a la Educación Superior. Los Sistemas de becas, créditos educativos, estímulos son factores que han permitido el acceso a hijos de familias pobres, generando de alguna manera la ruptura de la pobreza y un resultado social importante.

El porcentaje de becas otorgadas a los quintiles 1, 2 y 3 es mayor respecto al quintil 5 . Esto refleja acciones que permiten el acceso a la Educación Superior a los hijos de las familias pobres, que incluso reciben un bono del Estado y que nunca tuvieron la posibilidad remota de pensar en acceder a las Universidades o Escuelas Politécnicas.

Se evidencia también que las etnias indígenas y afroecuatorianas, han duplicado el acceso, gracias a la gratuidad de la Educación Superior, que en la Constitución es valorada como un derecho y un bien público, abriendo nuevas oportunidades para los pueblos y nacionalidades. Ahora más del $30 \%$ de estudiantes universitarios son indígenas y afroecuatorianos.

En la actualidad, Ecuador cuenta con Universidades y Escuelas Politécnicas con excelencia académica, reconocidas a nivel nacional e internacional. Sin embargo, también existe un número significativo de instituciones de Educación Superior, que se encuentran en proceso de acreditación para alcanzar los estándares establecidos de calidad educativa. Sin embargo, en la actualidad se evidencia la existencia de institutos técnicos y tecnológicos que forman parte de la Educación Superior, presentando un porcentaje muy bajo en relación con el tercer nivel de educación, en relación con otros países de América Latina.

Ante esta situación resalta la importancia de aplicar modelos de evaluación institucional direccionados a conocer las debilidades y fortalezas que presentan las instituciones de educación superior en busca de la calidad educativa en función de lograr la formación integral del recurso humano en el contexto ecuatoriano.

En función de las ideas expuesta, el objetivo de la presente investigación es: Describir la estructura y dinámica de aplicación de los modelos de evaluación institucional para la calidad educativa 


\section{Fundamento Teórico}

Evaluación institucional. Es importante destacar que en este espacio se plantean los aportes teóricos relacionados con la evaluación institucional, lo cual ha contribuido a la profundización de la temática enriqueciendo el acervo del conocimiento. En este sentido, Manzano (2015) expresa que la evaluación institucional es un proceso sistemático que valora, aprecia y estima la calidad de las instituciones evaluadas. Se trata pues de realizar un análisis de fortalezas y debilidades, un profundo estudio de las características que envuelven la realidad de una organización con el fin de definir una serie de planes de actuación para la mejora de la enseñanza. Vista así la evaluación descarta su carácter coercitivo y punitivo que se le acusa.

Finol de Franco y Col (2002) define la evaluación institucional como un proceso de negociación sistemático, participativo, de responsabilidad compartida entre todos los actores involucrados en el quehacer de una organización educativa, cuyo propósito es el mejoramiento continuo de los procesos académicos y administrativos, lo cual tiene como punto de referencia la realidad contextualizada de la institución, su dinámica de funcionamiento, así como las expectativas de las personas e instancias, dirigidas a tomar decisiones que contribuyan con el logro de la eficiencia y eficacia organizacional

Desde el punto de vista del Sistema de Evaluación y Acreditación (SEA), (2002), se expone que la evaluación institucional le da pertinencia al conocimiento de lo evaluado, más que su calificación; cuyo conocimiento deberá generar decisiones, las cuales estarán matizadas por el propósito específico de cada evaluación.

Para Romero y Triviño la evaluación institucional constituye

un conjunto de procedimientos y estrategias encaminados al compromiso con el cliente, la mejora continua, la sistematización en la resolución de problemas y el fortalecimiento del personal. Propone con fundamento teórico las acciones necesarias para reconducir las actuaciones hacia la mejora y abre vías de investigación futuras sobre las demandas y satisfacción del cliente, motivación e implicación de las personas y satisfacción de la comunidad en general (2006:190).

La Evaluación Institucional desde la perspectiva de la Comisión Nacional de Evaluación y Acreditación Universitaria CONEAU (1994) es un proceso que tiene por objeto el análisis de los logros y dificultades en el cumplimiento de la función 
institucional, así como también sugerir medidas para su funcionamiento. Abarca a las funciones de docencia, investigación y extensión.

De igual forma Vallone se entiende por evaluación institucional al proceso de recolección de información necesaria y el análisis que permite construir un saber acerca del establecimiento, diseñar estrategias y tomar decisiones" (2012, citando a Frigerio y Poggi, 1992). La evaluación institucional como proceso permite valorar la calidad educativa, constatando el estado real de los procesos académicos, organizacionales y comunitarios de las instituciones universitarias, lo cual implica la puesta en marcha de la autoevaluación (evaluación interna), la evaluación externa, con base a los resultados obtenidos de éstas se aspira a la acreditación de planes, programas y de la propia institución

\section{Calidad Educativa}

Leones (2018) explica que la calidad educativa es una construcción colectiva y una responsabilidad compartida por todos los involucrados en el hecho educativo y su evaluación debe asumir, entonces, un carácter eminentemente formativo. Un proceso de evaluación con carácter formativo, además de exhibir resultados, enfatiza la interpretación y comprensión en torno a los mismos, en términos de qué transmiten y qué muestran esos resultados, y acciona en consecuencia para la mejora educativa.

Por su parte, la UNESCO plantea que "La calidad de la educativa aún no está definida por circunstancias como: el lugar y el medio en que nace un niño, su sexo, los recursos económicos de sus padres, el idioma que habla y el color de su piel" (2010:35). En tal sentido promueve la educación de calidad como un derecho humano

De igual manera, Dias Sobrinho (2006) plantea que la calidad educativa es una construcción social, que varía según los intereses de los grupos de dentro y de fuera de las instituciones. En la consideración de Seibold (2000) manifiesta que la calidad educativa en sus inicios se entendió como un reformismo pedagógico basado en lo cognitivo, a partir del cambio de planes y prácticas pedagógicas. Luego, se introdujo el concepto de calidad total tomado desde el ámbito empresarial, estableciendo un destinatario de la labor educativa, en este caso el alumno, como un beneficiario o cliente. Pérez (2001:23); indica que la calidad educativa da cuenta de la relación entre los elementos claves del proceso educativo, apareciendo como un concepto teórico muy complejo con alto grado de abstracción cuyas dimensiones son la funcionalidad, la eficacia y la eficiencia, la relación 
que integra las dimensiones se hace a través de niveles o grados en un sistema determinado. A estas dimensiones en educación se suele agregar la dimensión equidad.

\section{Modelos para la Evaluación Institucional}

¿La evaluación ha sido objeto de discusión y análisis de cómo implementarla? ¿Para qué?, ¿Cuándo? generando diversas propuestas en el campo empresarial, centrado en la búsqueda de la excelencia, como proceso de mejora continua direccionado hacia la calidad total, a nivel mundial se reconoce la utilidad de esfuerzos concretados en modelos y reconocimiento de las empresas y/o organizaciones de calidad, destacando el premio de Deming de la calidad(1951), el certificado ISO 9000 (1970), el premio Malcolm Baldridge (1987), el EFQM (1991), así como los trabajos de Hurán, Ishikawa entre otros, los cuales han aportado fundamentos teóricos-referenciales para la calidad total, sirviendo de base sustantiva para el estudio, análisis y adecuación de dichos modelos al campo educativo

Para el desarrollo de la evaluación institucional en el ámbito de la educación superior, y en especial de las universidades a los fines de determinar la calidad de los procesos académicos, organizativos que indudablemente afectan de manera positiva o negativa la calidad del servicio que prestan a la sociedad se han aplicado una serie de modelos de evaluación entre los que destacan Modelo Malcolm Baldrige, Modelo EFQM, Modelo CINDA, Modelo de Gento Palacios, en el caso específico del Ecuador, se tiene a los órganos rectores encargadas de valorar la pertinencia, eficiencia y eficacia de las instituciones de educación superior caso: CONEA, CEAACES

Los modelos de Malcolm Baldrige y el EFQM, si bien no son modelos surgidos en el ámbito educativos tiene su aplicación en la misma, adecuando su estructuras y procesos a la dinámica organizacional de las instituciones universitarias a través de la autoevaluación y coevaluación, basada en las funciones sustantivas de éstas como son la docencia, investigación y relación con el entorno

\section{Modelo Malcolm Baldrige}

El modelo Malcolm Baldrige conocido como el modelo estadounidense para la gestión de la calidad total (GCT) constituye una guía hacia la Excelencia que proporciona a las organizaciones un enfoque integrado para la gestión del desempeño organizacional que mejore la eficacia y las capacidades de toda la organización, 
proporcione valor añadido a los clientes y grupos de interés, contribuyendo a la sostenibilidad y gestión del conocimiento de la organización.

En función de lo expuesto por Revilla (2004) la característica principal es que tiene una orientación a resultados. Plantea la necesidad de desarrollar alineamiento organizacional, desarrolla un enfoque en clientes y busca la focalización en todos los procesos, áreas y actividades clave del negocio.

Se ha considerado importante como un modelo clave en el proceso de reconvertir de manera estratégica a las organizaciones. Es utilizado con adaptaciones en todo el mundo. Actualmente tiene pertinencia en el sector privado y público, además de organizaciones sin fines de lucro. El modelo considera siete puntos clave, que permiten evaluar el sistema de gestión de la calidad, estos son:

- Liderazgo: La alta dirección debe establecer una visión orientada hacia el cliente, demostrar valores y ética organizacionales claros y visibles, y establecer altas expectativas para el personal balanceando las necesidades de todas las partes interesadas

- Planificación estratégica: Plantea para marcar el futuro camino, no exenta de cambios, las organizaciones tratan de fijar unas metas y marcar un sendero hacia ellas. Existen diferentes metodologías para lograr este reto

- Orientación al cliente y al mercado: El desempeño y la calidad son juzgados por los clientes de la organización, por lo cual deben tenerse en cuenta todas las características de los productos y servicios y todos los modos de acceso y apoyo que agregan valor a los clientes. Tal comportamiento conduce a la adquisición, satisfacción, preferencia, fidelidad y lealtad del cliente; referencias positivas y finalmente la sostenibilidad del negocio

- Información y análisis: Examina como la organización selecciona, recolecta, analiza, gestiona y mejora los activos de datos, información y conocimiento y como también evalúa el desempeño organizacional,

- Orientación hacia las personas: Es fundamental enfocarse al cliente, conocer sus necesidades y cubrirlas. Conocer la tendencia del mercado para operacionalizar una estrategia competitiva.

- Gestión de los procesos: Se tener conocimiento sobre la causa. La creación de un mapa claro de procesos con el que trabajar delimitará donde empieza y termina las acciones. Se debe tener un mapeo que refleje procedimientos de forma clara. 
- Resultados del negocio: Al terminar se debe fijar los resultados obtenidos, para valorar la balanza financiera, satisfacción de cliente, gestión con proveedores, competitividad en el mercado, entre otros. (Ver figura 1).

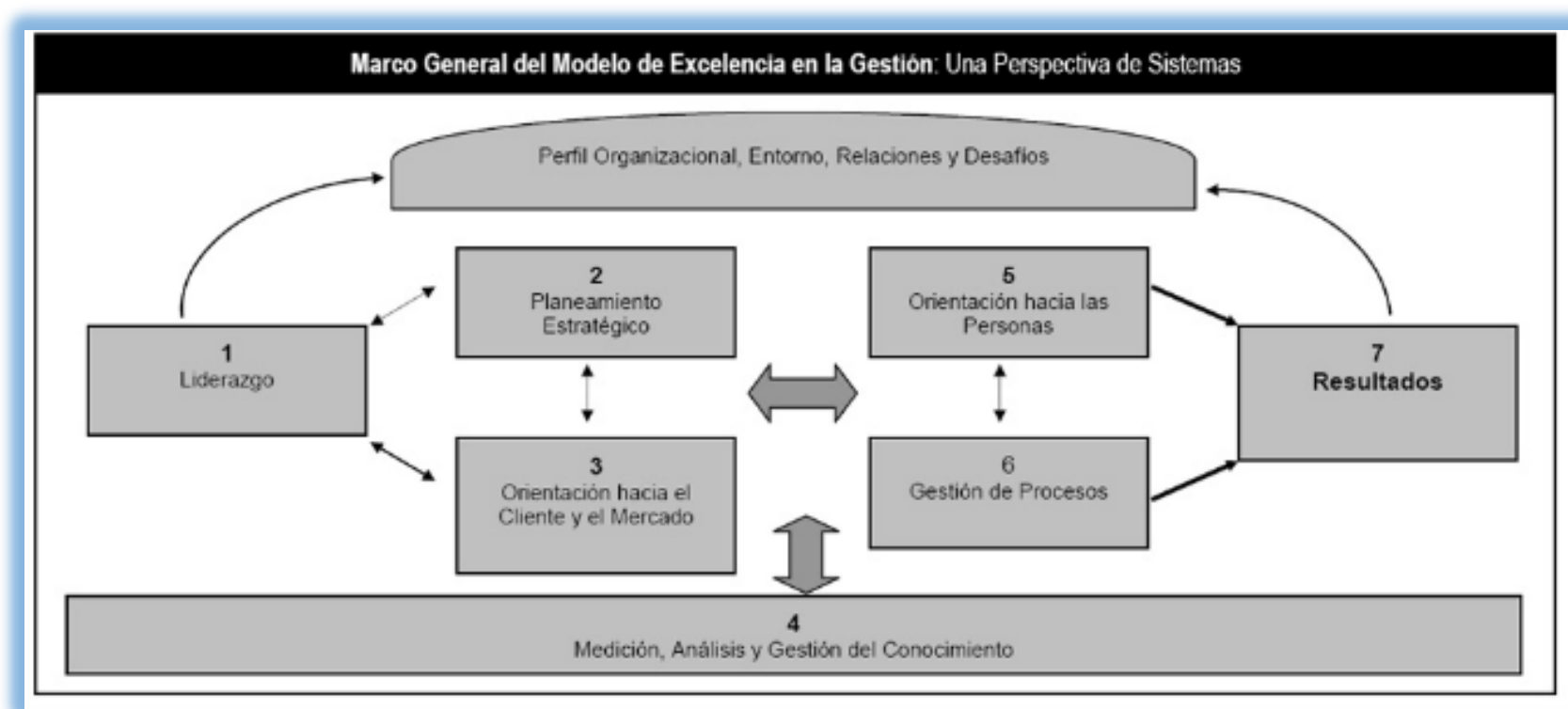

Figura 1: Modelo de Excelencia de Gestión. Fuente: Revilla (2004)

Es importante destacar que el Modelo Malcolm Baldrige de acuerdo a lo expuesto por Revilla (2004) planteo que en el año 1987, el congreso de los Estados Unidos promulgó la ley pública 100 - 107 estableciendo el Premio Nacional de Calidad Malcom Baldrige, otorgado anualmente a aquellas organizaciones que obtengan los más altos puntajes en los siguientes criterios que conforman el modelo Baldrige: Liderazgo, Información y análisis, Planificación estratégica, Gestión de los Recursos Humanos (RR HH), Garantía de calidad de productos y servicios, Resultados de calidad y Satisfacción del cliente. Todo esto se plantea porque ofrece la versatilidad de poder adaptarse a la escuela, y establecer los estándares que se desean lograr en función de las metas establecidas, sin que le sean impuestas directamente. Además, presentan como valor agregado importante de este modelo las verificaciones anuales de sus dimensiones y estándares. El modelo Malcolm Baldrige está vigente desde hace 20 años en EEUU, en Japón y en 22 países iberoamericanos, incluyendo España y Portugal, para la evaluación de organizaciones educativas que desean demostrar su idoneidad para ofrecer servicios de excelencia 


\section{Modelo EFQM}

El modelo EFQM (European Foundation for Quality Management) según Parra, Villa y Restrepo (2009) es un modelo que nació por iniciativa de 14 de las mayores empresas europeas que constituyeron en 1988 la Fundación Europea para la Gestión de la Calidad, la cual en 1991 creó el Premio Europeo de Excelencia Empresarial, el cual considera cuatro categorías, entre las cuales se incluye el "Premio Europeo a la Calidad para Pequeñas y Medianas Empresas”. El modelo de acuerdo con Carrión (2006) consta de nueve criterios, que permiten evaluar el estado de la organización en cuanto a su gestión de la calidad. Se fundamenta en la premisa de que "la satisfacción del cliente, la satisfacción de los empleados y un impacto positivo en la sociedad se consiguen mediante el liderazgo en política y estrategia, una acertada gestión de personal, el uso eficiente de los recursos y una adecuada definición de los procesos, lo que conduce finalmente a la excelencia de los resultados empresariales". Los nueve criterios del modelo son agrupados en dos grandes bloques llamados Agentes Facilitadores y Resultados; los primeros analizan la forma en que la empresa planea y ejecuta sus actividades, y los últimos se refieren a los logros alcanzados por ella, tal como se muestra en la figura 2. Los Agentes Facilitadores hacen alusión a la gestión sobre el liderazgo, el personal, las políticas y estrategias, las alianzas y recursos y los procesos. En lo referente a los Resultados, el modelo considera cuatro áreas en las cuales mide el impacto provocado por la actividad de la empresa, éstas son: resultados en las personas, en los clientes, en la sociedad y resultados clave. 


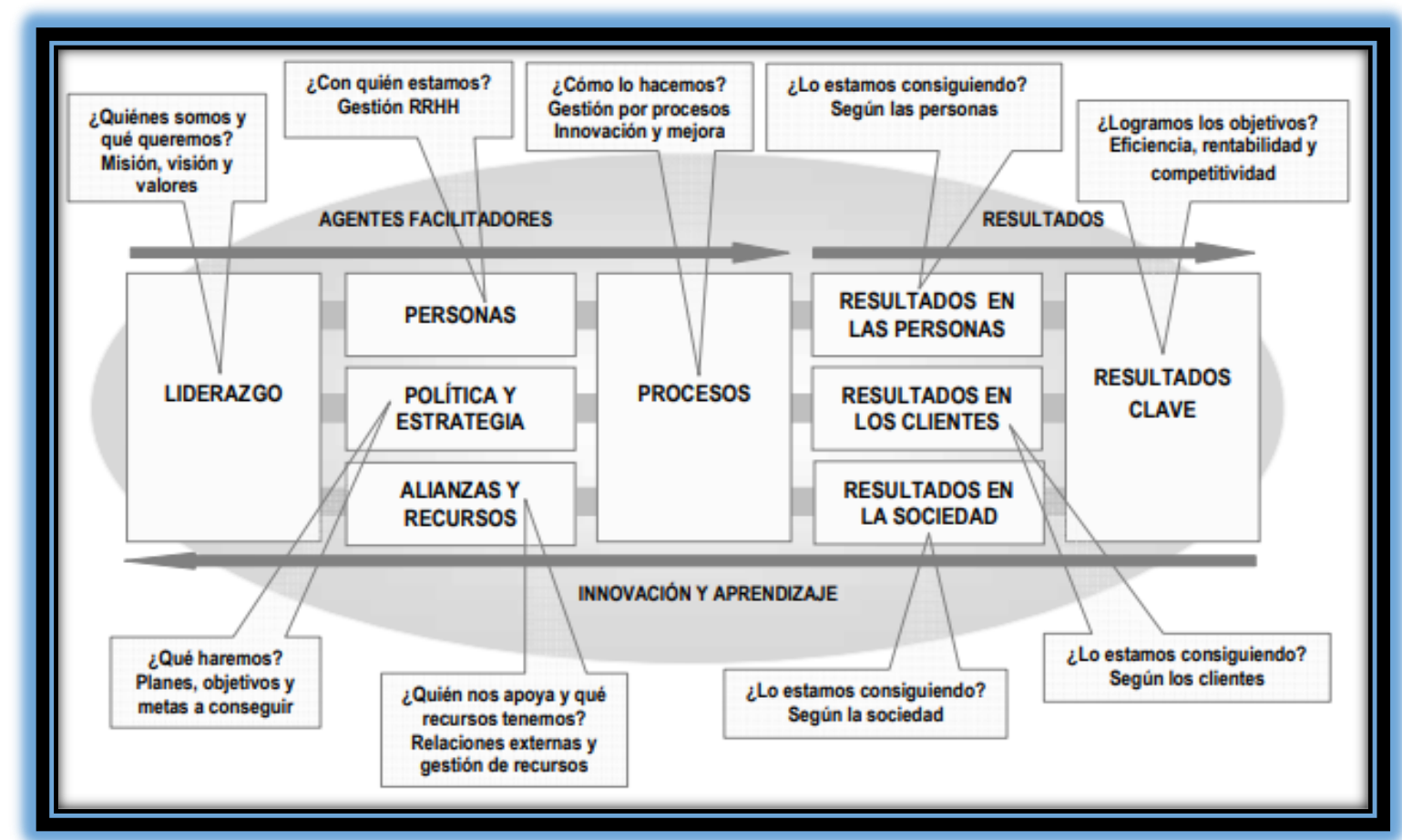

Figura 2: Estructura del modelo EFQM, de la Fundación Europea para la Gestión de la Calidad. Fuente: Carrión (2006)

Es importante lo expuesto por Martínez y Rioperez (2005) quienes plantean que las administraciones educativas, los sistemas educativos y las instituciones educativas públicas y privadas participan del interés por el movimiento de la calidad total, para el logro de la excelencia, a través de la implicación de todos los miembros de la institución, en sus distintos niveles de responsabilidad, en los procesos de gestión y autoevaluación para la mejora y rendimiento de cuentas a la sociedad.

La Comisión Europea, desde 1997, promueve la utilización del Modelo EFQM para la evaluación de instituciones educativas, y son muchas las administraciones educativas públicas que lo han adaptado. En España, desde esa fecha, el Club Gestión de Calidad (CGC), representante en España de EFQM, con el Instituto de Técnicas de Estudio de la Confederación Española de Centros de Enseñanza (ITE, CECE) y el Ministerio de Educación, Cultura y Deporte (MECD), hicieron una adaptación del modelo para su aplicación en los Centros Educativos, actualizada en el 2001 que ha sido revisada en años posteriores.

La finalidad del modelo aplicado al ámbito educativo es conocer la realidad del centro mediante la autoevaluación, y reflexionar sobre ella, lo que posibilitará elaborar planes y estrategias de mejora. Estas dos características, junto con la implicación de todos en el proceso de autoevaluación y la toma de decisiones, se consideran factores que 
inciden en la mejora de la calidad de los servicios que el Centro Educativo presta a la sociedad.

El modelo define nueve factores críticos para el buen funcionamiento de la organización a los que denomina criterios, fundamentados en elementos o indicadores. La comprobación sistemática de la existencia o no de dichos indicadores en la institución, como procedimiento para la autoevaluación, permite tener un conocimiento del funcionamiento del centro basado en hechos, con el fin de elaborar proyectos y planes de mejora en coherencia con las necesidades detectadas. La utilización de la autoevaluación para la recogida de información, como herramienta de mejora continua, permitirá avanzar en el logro de la política y estrategia del centro, concretados, entre otras cosas, en los objetivos de aprendizaje y formación de los alumnos, teniendo en cuenta la formación del personal y los recursos necesarios hacia la excelencia.

\section{Modelo CINDA}

El Centro Interuniversitario de Desarrollo (CINDA) por la Universidad Carlos III de Madrid (UC3M) (2017) es una corporación internacional sin fines de lucro integrada por importantes universidades de América Latina y Europa. Este centro es pionero en el establecimiento de redes de colaboración entre instituciones de educación superior, un trabajo que ha llevado a cabo ininterrumpidamente desde hace más de treinta años. CINDA, en conjunto con las universidades integradas al sistema, ha explorado temas centrales sobre política y gestión universitaria con el interés de poner el conocimiento acumulado al servicio de las políticas públicas y de las instituciones correspondientes.

CINDA (1994) ha centrado su quehacer en dos modalidades principales. La primera consiste en el análisis y reflexión sistemática acerca del desarrollo de la educación superior y de sus implicaciones en diversos ámbitos. De esa forma, contribuye a la sistematización de experiencias, a la revisión de enfoques y perspectivas sobre la educación superior y a la difusión del conocimiento, con la finalidad de promover el diseño y desarrollo de políticas y mecanismos de gestión y trabajo en las instituciones de educación superior. La segunda se materializa en el impulso de actividades concretas de cooperación académica, aprovechando modalidades de cooperación horizontal. Este tipo de colaboración entre universidades que enfrentan problemas y desafíos comunes complementa exitosamente las iniciativas de carácter vertical a que se refiere la primera modalidad mencionada. 
CINDA contempla seis (6) dimensiones: relevancia, efectividad eficiencia, eficacia, recursos y procesos; cada una de estas dimensiones contienen criterios a través de los cuales es posible aproximarse a las dimensiones y su evaluación. El modelo en cuestión se sustenta en las siguientes premisas: Calidad no es un concepto absoluto sino relativo, ¿el referente de calidad está establecido en la propia institución cuando define qué va a hacer? Es decir, su misión, objetivos, metas y estrategias. Por otra parte, el modelo CINDA (1970) hace énfasis en la autoevaluación como proceso interno de participación de los miembros de la institución

\section{Modelo de Gento Palacios}

El Modelo de Calidad para las Instituciones Educativas del Dr. Gento Palacios desarrollado por el autor en 1996, según expone Vivas (1999) pretende ofrecer una visión objetiva, integral y ponderada de lo que deben ser las instituciones para ser consideradas de calidad. Parte del enfoque de totalidad o globalidad, por cuanto considera que en el análisis de la calidad deben considerarse todos los componentes que intervienen en el proceso educativo y sus mutuas relaciones, involucrar a todos los ámbitos posibles y abarcar tanto los procesos como los resultados de la educación.

El modelo es estructurado en torno a dos componentes: los indicadores y los predictores. Los indicadores son aquellos rasgos configurativos que colocarán de manifiesto el grado de calidad alcanzado y evidencian la calidad de un centro. Por su parte, los predictores son los factores o características que han de reunir las instituciones educativas para lograr niveles aceptables de calidad; como su nombre lo indica, los predictores permiten predecir que dicha calidad va a producirse.

Dentro del modelo propuesto, se considera como identificadores de la calidad el producto educativo, la satisfacción de los propios estudiantes, la satisfacción del personal que trabaja en la institución y el efecto de impacto de la educación alcanzada. Por su parte, los componentes predictores hacen referencia a la disponibilidad de medios personales y materiales, organización de la educación y gestión de los recursos enmarcados en el liderazgo pedagógico. Ver figura 3. 


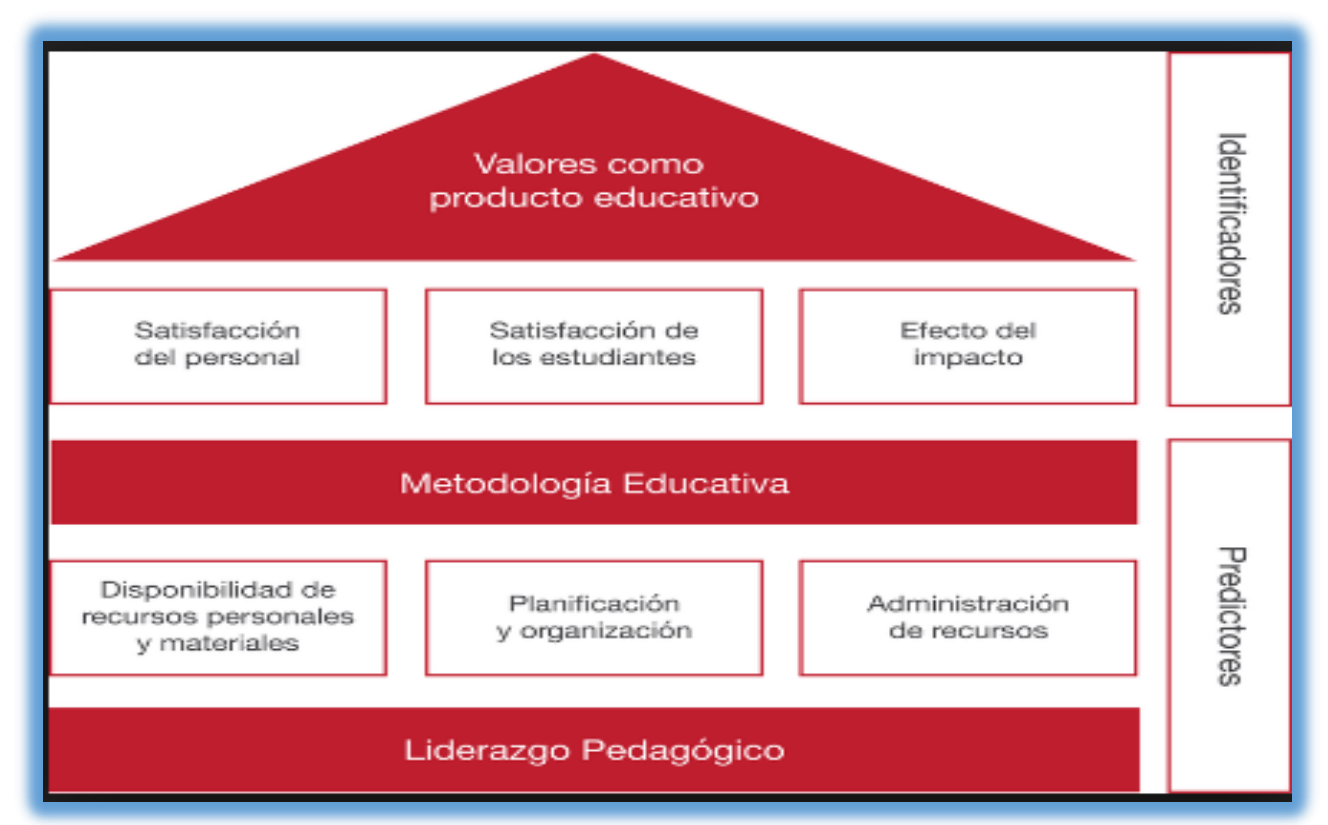

Figura 3: Modelo de Calidad Total para las Instituciones Educativas de Gento Palacios. Fuente: Gento Palacios (2002)

Se destaca los expuesto por Gento, (2002:243) al afirmar que la "evaluación será un elemento potenciador del replanteamiento constante de todo el proyecto en sus diferentes fases".

El Jaime y Medina afirman que:

La evaluación de un proyecto no debe, pues, constituir una acción puntual llevada a cabo en un solo momento de su gestión o desarrollo; por el contrario, ha de ser inherente al desarrollo del propio proyecto en sus diferentes fases, y debe estar permanentemente desde antes, incluso, de su implantación. En realidad, formará parte de cada una de las fases de desarrollo, de modo que pueda analizarse cada una de ellas y pueda llegarse al reconocimiento de los factores o causas de los éxitos y de las fallas, así como de las posibles interacciones de estos (2010:4).

\section{Modelos asumidos por el CONEA, CEAACES}

Las instituciones ecuatorianas, tienen una variada experiencia en evaluación, aunque diferente a la institucional. Es el caso de la evaluación curricular, evaluación al docente, evaluación de los aprendizajes, evaluación de proyectos de investigación, entre otras.

El Sistema Nacional de Evaluación y Acreditación de la Educación Superior (SEAES), está integrado por todas las instituciones de educación superior, de las cuales, 62 son universidades y escuelas politécnicas, 277 institutos superiores técnicos y 
tecnológicos, el Consejo Nacional de Evaluación y Acreditación de la Educación Superior (CONEA), como organismo acreditador; el componente legal que lo integra la Constitución Política de la República, la Ley Orgánica de Educación Superior (LOES), el Reglamento General del SEAES; y, por el componente técnico que lo integran las características y estándares de calidad, guías de autoevaluación, Manual de Evaluación Externa y otros instructivos y documentos técnicos.

Tomando en cuenta el mencionado Modelo del Consejo Nacional de Evaluación y Acreditación (CONEA) (2003) se destaca que se encuentran tipificados en el Art. 90 de la Ley Orgánica de Educación Superior (LOES), comprende tres etapas: la Autoevaluación, la Evaluación Externa y la Acreditación. Cada una de ellas tiene su propia caracterización y metodología que orientan su aplicación y desarrollo. La autoevaluación es en sí una manera de medir las actividades que la universidad realiza a través de la docencia e investigación y que estas estén en conexión con la colectividad, función básica de toda universidad. Una vez que se inició el proceso de autoevaluación, se efectuará la fase de socialización de la información, para lo cual en primera instancia se formará equipos de trabajo en las escuelas respectivas. La participación de docentes, investigadores, como estudiantes de gestión productiva y desde luego con la implicación del director de la escuela, permitirá que trabajen conjuntamente en la dimensión específicamente de la docencia y formación de recursos humanos.

En este orden de ideas, en los últimos años las universidades del Ecuador han demostrado un creciente interés por conocer y profundizar el tema de la evaluación, aspecto verificable por la organización y participación en una variedad de eventos de capacitación, tanto en el país como en el exterior.

Por otra parte, el artículo 171 de la Ley Orgánica de Educación Superior (LOES) vigente en el Ecuador, establece que el Consejo de Evaluación, Acreditación y Aseguramiento de la Calidad de la Educación Superior (CEAACES) es el organismo público técnico, con personería jurídica y patrimonio propio, con independencia administrativa, financiera y operativa.

Funciona en coordinación con el Consejo de Educación Superior. Tiene facultad regulatoria y de gestión. No puede conformarse por representantes de las instituciones objeto de regulación ni por aquellos que tengan intereses en las áreas que vayan a ser reguladas. 
De conformidad con el artículo 174 de la LOES, son funciones del Consejo de Evaluación, Acreditación y Aseguramiento de la Calidad de la Educación Superior (SEAACES):

- Planificar, coordinar y ejecutar las actividades del proceso de evaluación, acreditación, clasificación académica y aseguramiento de la calidad de la educación superior;

- Aprobar la normativa para los procesos de evaluación, acreditación, clasificación académica y aseguramiento de la calidad de las instituciones del Sistema de Educación Superior, programas y carreras, bajo sus distintas modalidades de estudio;

- Aprobar la normativa para los procesos de la autoevaluación de las instituciones, los programas y carreras del Sistema de Educación Superior;

- Aprobar la normativa en la que se establecerá las características, criterios e indicadores de calidad y los instrumentos que han de aplicarse en la evaluación externa;

- Elaborar la documentación técnica necesaria para la ejecución de los procesos de autoevaluación, evaluación externa, acreditación y clasificación académica;

- Aprobar el Código de Ética que regirá para los miembros del Consejo, Comité Asesor, y los funcionarios y las y los Servidores del Consejo de Evaluación, Acreditación y Aseguramiento de la Calidad de la Educación Superior, y para los evaluadores externos.

- Aprobar el reglamento de selección de los evaluadores externos especializados nacionales o internacionales;

- Calificar, a los evaluadores externos especializados, nacionales o internacionales, para la ejecución de procesos de evaluación externa, acreditación y clasificación académica de las instituciones del Sistema de Educación Superior, las carreras y programas;

- Vigilar que los procesos de evaluación interna y externa se realicen de conformidad con las normas y procedimientos que para el efecto se establezcan y garantizar que sus resultados sean fruto de una absoluta independencia, imparcialidad y ética con la labor desempeñada;

- Resolver sobre los informes y recomendaciones derivados de los procesos de evaluación, acreditación y clasificación académica; 
- Otorgar certificados de acreditación institucional así como para programas y carreras, a las instituciones de educación superior y unidades académicas que hayan cumplido con todos los requisitos exigidos para el efecto. Este certificado de acreditación tendrá una vigencia de cinco años y no podrá estar condicionado;

- Determinar la suspensión de la entrega de fondos a las instituciones de educación superior en la parte proporcional cuando una o más carreras o programas no cumplan los estándares establecidos, e informar al Consejo de Educación Superior para su ejecución;

- Establecer un sistema de categorización de instituciones, programas y carreras académicas;

- Divulgar ampliamente los resultados de los procesos de evaluación externa, acreditación y clasificación académica con el propósito de orientar a la sociedad ecuatoriana sobre la calidad y características de las instituciones, programas y carreras del sistema de educación superior;

- Asesorar al Ministerio de Educación en la implementación y ejecución de la evaluación y acreditación para la educación básica y media, con fines de articulación con la educación superior;

- Presentar anualmente informe de sus labores a la sociedad ecuatoriana, al Presidente de la República, a la Asamblea Nacional, y al Consejo de Participación Ciudadana y Control Social;

- Firmar convenios con instituciones de educación superior para la formación y capacitación de los evaluadores a fin de profesionalizar esta labor;

- Establecer convenios con entidades internacionales de evaluación y acreditación de la educación superior para armonizar procesos y participar de redes; propiciar la evaluación y reconocimiento internacional de este organismo y de las instituciones de educación superior ecuatorianas;

- Ejecutar prioritariamente los procesos de evaluación, acreditación y clasificación académica de programas y carreras consideradas de interés público;

- Diseñar y aplicar la Evaluación Nacional de Carreras y Programas de último año, así como procesar y publicar sus resultados;

- Elaborar los informes que le corresponden para la creación y solicitud de derogatoria de la Ley, decreto Ley, decreto, convenio o acuerdo de creación de universidades y escuelas politécnicas; 
- Elaborar los informes que le corresponden para la creación y extinción de institutos superiores técnicos, tecnológicos, pedagógicos, de artes y conservatorios superiores;

- Elaborar y aprobar la normativa que regule su estructura orgánica funcional, y elaborar su presupuesto anual;

- Elaborar los informes de suspensión de las instituciones de educación superior que no cumplan los criterios de calidad establecidos, y someterlos a conocimiento y resolución del Consejo de Educación Superior;

- Realizar seguimiento sobre el cumplimiento de los aspectos académicos y jurídicos de las Instituciones de Educación Superior; y, las demás que determine la LOES y sus reglamentos.

\section{Orientación metodológica}

Esta investigación se circunscribe a las investigaciones de tipo documental tal y como lo afirma Arias “es un proceso basado en la búsqueda, recuperación, análisis, crítica e interpretación de datos secundarios, es decir, los obtenidos y registrados por otros investigadores en fuentes documentales: impresas, audiovisuales o electrónicas" (2012:27). Para lo cual se siguió el siguiente procedimiento:

1. Revisión documental: Textos, leyes, reglamentos e informes, relacionados con la evaluación institucional.

2. Con base a la revisión documental y aplicación de análisis de contenido se elaboró el cuadro 1 donde se presenta la definición, estructura y el respectivo comentario en relación a los autores generadores de modelos de evaluación, así como órganos e instituciones

\section{Hallazgos}

En el cuadro 1 se presentan los modelos de Evaluación estudiados en la presente investigación, tales como Malcolm Baldrige (1987), Modelo EFQM (1980), Modelo CINDA (1970), Modelo Gento Palacios (1996) y los Modelos asumidos por el CONEA, CEAACES (2003) para lo cual se expresa la definición y las estructuras base que se aplican para efectuar el proceso evaluativo 


\section{Cuadro 1}

\section{Modelos de Evaluación}

\begin{tabular}{|c|c|c|c|}
\hline Modelo & Definición & Estructura & Comentario \\
\hline $\begin{array}{l}\text { Modelo Malcolm } \\
\text { Baldrige(1987) }\end{array}$ & $\begin{array}{l}\text { Plantea la necesidad de } \\
\text { desarrollar } \\
\text { alineamiento } \\
\text { organizacional, } \\
\text { desarrolla un enfoque } \\
\text { en clientes y busca la } \\
\text { focalización en todos } \\
\text { los procesos, áreas y } \\
\text { actividades clave del } \\
\text { negocio. Revilla (2004) }\end{array}$ & $\begin{array}{l}\text { Establece siete } \\
\text { criterios importantes: } \\
\text { - } \quad \text { Liderazgo } \\
\text { - } \quad \text { Planificación } \\
\text { - } \quad \text { Orientación al } \\
\text { cliente } \\
\text { - } \quad \text { Información y } \\
\text { análisis } \\
\text { - Orientación a las } \\
\text { - } \text { gersonas } \\
\text { - } \text { procestión de los } \\
\text { Resultados }\end{array}$ & $\begin{array}{l}\text { Es utilizado con } \\
\text { adaptaciones en todo el } \\
\text { mundo. Actualmente } \\
\text { tiene pertinencia en el } \\
\text { sector privado y público, } \\
\text { además de } \\
\text { organizaciones sin fines } \\
\text { de lucro, tal es el caso de } \\
\text { las instituciones } \\
\text { educativas cuya filosofía } \\
\text { de gestión difiere de las } \\
\text { empresas comerciales e } \\
\text { industriales cuyo } \\
\text { proceso y resultados } \\
\text { definen la calidad en } \\
\text { términos de } \\
\text { productividad y } \\
\text { competitividad, y } \\
\text { marcando diferencias } \\
\text { con las organizaciones } \\
\text { educativas generadoras } \\
\text { de formación y y } \\
\text { capacitación del talento } \\
\text { humano }\end{array}$ \\
\hline $\begin{array}{c}\text { Modelo EFQM } \\
\text { (1980) }\end{array}$ & $\begin{array}{l}\text { Permiten evaluar el } \\
\text { estado de la } \\
\text { organización en cuanto } \\
\text { a su gestión de la } \\
\text { calidad. Carrion (2006) }\end{array}$ & $\begin{array}{l}\text { Establece nueve } \\
\text { criterios del modelo } \\
\text { que se agruparon en } \\
\text { dos grandes bloques } \\
\text { llamados Agentes } \\
\text { Facilitadores y } \\
\text { Resultados; los } \\
\text { primeros analizan la } \\
\text { forma en que la } \\
\text { empresa planea y } \\
\text { ejecuta sus actividades, } \\
\text { y los últimos se } \\
\text { refieren a los logros } \\
\text { alcanzados por ella. } \\
\text { Los Agentes } \\
\text { Facilitadores hacen } \\
\text { alusión a la gestión } \\
\text { sobre el liderazgo, el } \\
\text { personal, las políticas } \\
\text { y estrategias, las } \\
\text { alianzas y recursos y } \\
\text { los procesos. En lo } \\
\text { referente a los } \\
\text { Resultados, el modelo } \\
\text { considera cuatro áreas } \\
\text { en las cuales mide el } \\
\text { impacto provocado por } \\
\text { la actividad de la } \\
\text { empresa, éstas son: } \\
\text { resultados en las }\end{array}$ & $\begin{array}{l}\text { Es un modelo que nació } \\
\text { por iniciativa de } 14 \text { de } \\
\text { las mayores empresas } \\
\text { europeas que } \\
\text { constituyeron en } 1988 \text { la } \\
\text { Fundación Europea para } \\
\text { la Gestión de la Calidad, } \\
\text { la cual en } 1991 \text { creó el } \\
\text { Premio Europeo de } \\
\text { Excelencia Empresarial, } \\
\text { el cual considera cuatro } \\
\text { categorías, entre las } \\
\text { cuales se incluye el } \\
\text { "Premio Europeo a la } \\
\text { Calidad para Pequeñas y } \\
\text { Medianas Empresas". }\end{array}$ \\
\hline
\end{tabular}




\begin{tabular}{|c|c|c|c|}
\hline & & $\begin{array}{l}\text { personas, en los } \\
\text { clientes, en la sociedad } \\
\text { y resultados clave. }\end{array}$ & \\
\hline $\begin{array}{c}\text { Modelo CINDA } \\
\qquad(1994)\end{array}$ & $\begin{array}{l}\text { Establece redes de } \\
\text { colaboración entre } \\
\text { instituciones de } \\
\text { educación superior. } \\
\text { (UC3M) (2017) }\end{array}$ & $\begin{array}{l}\text { Ha centrado su } \\
\text { quehacer en el análisis } \\
\text { y reflexión sistemática } \\
\text { acerca del desarrollo de } \\
\text { la educación superior y } \\
\text { de sus implicaciones en } \\
\text { diversos ámbitos. } \\
\text { Identifica seis (6) } \\
\text { dimensiones: } \\
\text { relevancia, } \\
\text { efectividad } \\
\text { eficiencia, eficacia, } \\
\text { recursos } \\
\text { procesos; cada una } \\
\text { de } \\
\text { dimensiones estas } \\
\text { contienen criterios }\end{array}$ & $\begin{array}{l}\text { Es pionero en el } \\
\text { establecimiento de redes } \\
\text { de colaboración entre } \\
\text { instituciones de } \\
\text { educación superior, un } \\
\text { trabajo que ha llevado a } \\
\text { cabo } \\
\text { ininterrumpidamente } \\
\text { desde hace más de } \\
\text { treinta años. Considera } \\
\text { la importancia de la } \\
\text { autoevaluación } \\
\text { mediante la } \\
\text { participación de los } \\
\text { miembros de la } \\
\text { institución }\end{array}$ \\
\hline $\begin{array}{c}\text { Modelo de Gento } \\
\text { Palacios } \\
(\mathbf{1 9 9 6 )}\end{array}$ & $\begin{array}{l}\text { Ofrecer una visión } \\
\text { objetiva, integral y } \\
\text { ponderada de lo que } \\
\text { deben ser las } \\
\text { instituciones para ser } \\
\text { consideradas de } \\
\text { calidad. Vivas (1999) }\end{array}$ & $\begin{array}{lr}\text { Establece } & \text { los } \\
\text { componentes } & \\
\text { identificadores } & \text { y } \\
\text { predictores para la } \\
\text { evaluación. } & \text { Los } \\
\text { identificadores } & \text { son } \\
\text { aquellos } & \text { rasgos } \\
\text { configurativos } & \text { que } \\
\text { colocarán } & \text { de } \\
\text { manifiesto el grado de } \\
\text { calidad alcanzado y } \\
\text { evidencian la calidad } \\
\text { de un centro. Por su } \\
\text { parte, los predictores } \\
\text { son los factores o } \\
\text { características que han } \\
\text { de reunir las } \\
\text { instituciones } \\
\text { educativas para lograr } \\
\text { niveles aceptables de } \\
\text { calidad; como su } \\
\text { nombre lo indica, los } \\
\text { predictores permiten } \\
\text { predecir que dicha } \\
\text { calidad va a producirse }\end{array}$ & $\begin{array}{l}\text { Es un modelo que aplica } \\
\text { a las instituciones } \\
\text { educativas bajo una } \\
\text { concepción holística y } \\
\text { sistémica, donde la } \\
\text { calidad está } \\
\text { determinada por la } \\
\text { conjunción de los } \\
\text { elementos que } \\
\text { participan en la } \\
\text { educación de manera } \\
\text { dinámica }\end{array}$ \\
\hline $\begin{array}{c}\text { Modelos } \\
\text { asumidos por el } \\
\text { CONEA, } \\
\text { CEAACES } \\
(2003)\end{array}$ & $\begin{array}{l}\text { CONEA: Es el } \\
\text { Consejo Nacional de } \\
\text { Evaluación y } \\
\text { Acreditación de la } \\
\text { Educación Superior que } \\
\text { se encarga de los } \\
\text { estándares de calidad } \\
\text { en las } \\
\text { Instituciones } \\
\text { Universitarias } \\
\text { CONEA(2003) } \\
\text { CEAACES: } \\
\text { Aseguramiento de la }\end{array}$ & $\begin{array}{l}\text { CONEA: Comprende } \\
\text { tres etapas: la } \\
\text { Autoevaluación, la } \\
\text { Evaluación Externa y } \\
\text { la Acreditación. Cada } \\
\text { una de ellas tiene su } \\
\text { propia caracterización } \\
\text { y metodología que } \\
\text { orientan su aplicación } \\
\text { y desarrollo. } \\
\text { CEAACES: Planifica, } \\
\text { coordina y ejecuta las } \\
\text { actividades del proceso }\end{array}$ & $\begin{array}{l}\text { CONEA: Se encuentran } \\
\text { tipificados en el Art. } 90 \\
\text { de la Ley Orgánica de } \\
\text { Educación Superior } \\
\text { (LOES) } \\
\text { CEAACES: Funciona } \\
\text { en coordinación con el } \\
\text { Consejo de Educación } \\
\text { Superior. Tiene facultad } \\
\text { regulatoria y de gestión. } \\
\text { No puede conformarse } \\
\text { por representantes de } \\
\text { las instituciones objeto }\end{array}$ \\
\hline
\end{tabular}




\begin{tabular}{|l|l|l|l|}
\hline & Calidad de la & de evaluación, & de regulación ni por \\
& Educación Superior que & acreditación, & aquellos que tengan \\
es el organismo público & clasificación & intereses en las áreas \\
& técnico, con personería & académica y & que vayan a ser \\
& jurídica y patrimonio & aseguramiento de la & reguladas \\
& propio, con & calidad de la educación & \\
independencia & superior. & \\
& administrativa, & & \\
financiera y operativa. & & \\
\hline
\end{tabular}

Fuente: Bermeo Paredes (2019)

\section{Reflexiones Finales}

La evaluación institucional para la calidad educativa constituye un constructo complejo, ya que en su planificación y desarrollo convergen diferentes factores externos e internos, además de los tipos de evaluación que deben aplicarse a los fines de valorar las funciones sustantivas de las instituciones universitarias, tal es el caso de las universidades Ecuatorianas, las cuales de acuerdo a la Ley Orgánica de Educación Superior se plantea la necesidad de fomentar una educación de calidad, donde destaca la formación del talento humano con las competencias requeridas por la sociedad globalizada, donde las tecnologías de la comunicación e información juega un papel de primer orden logrando trascender el espacio regional, nacional en pro de la internacionalización

Por ello, quienes dirigen las instituciones de educación superior y por supuestos los entes del Ministerio de Educación deben establecer una dirección estratégica que coadyuve a satisfacer las expectativas de los usuarios directos e indirectos y general de toda la sociedad. Para ello, se plantea la aplicación de modelos de evaluación tales como lo referenciados en el cuadro 1, modelo de Modelo Malcolm Baldrige (1987), Modelo EFQM (1980), Modelo CINDA (1994), Modelo de Gento Palacios (1996), cada uno de los modelos analizados presentan una estructura y dinámica de aplicación que deben ser adaptadas la realidad de las instituciones. En el caso del CONEA y el CEAACES como organismos rectores en la educación Ecuatoriana, plantean el aseguramiento de la calidad educativa mediante procesos de planificación y organización de la autoevaluación, evaluación externa y acreditación 


\section{Referencias Bibliográficas}

Alfaro, A y Pérez, M (2012). La evaluación institucional como factor de calidad en la educación superior. Propuesta de un sistema de evaluación. Primer Congreso Virtual Internacional sobre .Innovación Pedagógica y Praxis Educativa. Tomado el 7 de diciembre de 2018. Disponible en: https://dialnet.unirioja.es/servlet/libro?codigo $=535470$

Arias, F (2012). El proyecto de investigación. Editorial Episteme. Caracas. Venezuela.

Ávila, C y Ávila, O (2014). Modelo Malcom Baldrige en la creación de una escuela de formación musical como alternativa para el aprovechamiento del tiempo libre en la UPZ castilla. Tesis. Universidad Libre. Bogotá.

Carrión, A (2006). El modelo EFQM: más allá de ISO 9000. IB Revista de la Información Básica, vol. 1, No. 1

Centro Interuniversitario de Desarrollo (CINDA, 1994). Manual de Autoevaluaciones para Instituciones de Educación Superior. Pautas y Procedimientos. Santiago de Chile

CONEA (2017). Informe sobre calidad universitaria. Tomado el día 2 de enero de 2018. Disponible en: http://ecuadoruniversitario.com/noticias/noticias-de-interesgeneral/el-informe-del-conea-es-el-antecedente-de-la-evaluacion-que-lleva-a-cabo-elceaaces/

CONEAU (1994). Comisión Nacional de Evaluación Acreditación Universitaria. Tomado el día 12 de Noviembre de 2018. Disponible en:

https://www.coneau.gob.ar/?page id=124

Días Sobrinho, J. (2006). Acreditación de la Educación Superior en América Latina y el Caribe. En La Educación Superior en el Mundo 2007.Acreditación para la garantía de la calidad: ¿Qué está en juego?, Madrid.Barcelona: Global University Network for Innovation (GUNI/UNESCO,Ediciones Mundi-Prensa

Finol de Franco, M; Pelekais, C; Govea de Guerrero, M y Vallejo, R(2002). La Evaluación Institucional como Proceso de Optimización de la Gestión del Gerente Educativo. Ediciones ASTRODATA. Maracaibo, Venezuela

Gento Palacios, S (2002). Implementación de la Calidad Total en Instituciones de Educativas. Estudios UNED. Madrid. España

Jaime, M y Medina, M (2010). Modelo de calidad para las IES. 7mo Congreso Internacional de Educación Superior. México.

Leones, M (2018). La calidad de la educación es una construcción colectiva y una responsabilidad compartida. Oficina Regional de Educación de la UNESCO para América Latina y el Caribe. Tomado el 23 de noviembre de 2018. Disponible en: 
http://www.unesco.org/new/es/santiago/press-room/newsletters/newsletter-laboratoryfor-assessment-of-the-quality-of-education-llece/n14/mariela-leones/

Ley Orgánica de Educación Superior (LOES) (2010). Registro Oficial Suplemento 298. Asamblea Nacional. Quito. Ecuador.

Manzano, M (2015). Evaluación institucional: aproximación teórica conceptual. Tomado el día 14 de noviembre de 2018. Disponible en:

https://www.uls.edu.sv/sitioweb/component/k2/item/127-evaluacion-institucionalaproximacion-teorica-conceptual

Martínez, C y Rioperez, N (2005). El modelo de excelencia en la EFQM y su aplicación para la mejora de la calidad de los centros educativos. Facultad de Educación. UNED

Muñoz, M (2003). "El modelo EFQM como guía para la mejora y la excelencia, Foro por la Excelencia”. Qualitat Actual, , pp. 51-54.

Pérez, R. (2001). Hacia una educación de calidad. Madrid: Narcea

QAEC (2018). Modelo de Excelencia de Malcolm Baldrige. Tomado el día 12 de noviembre de 2018. Disponible en: https://www.aec.es/web/guest/centroconocimiento/malcolm-baldrige

Revilla, D (2004). La evaluación institucional: una estrategia válida para la gestión escolar. Volumen 3 No 24. Revista de Educación. Pontificia Universidad Católica del Perú. Perú

Romero Godoy, M. P., y Triviño García, M. Á. (2006). La evaluación en la universidad: un proceso de aprendizaje en las organizaciones universitarias. XXI: Revista de Educación, 8, 189-200.

Sistema de Evaluación y Acreditación de las Universidades Nacionales (SEA, 2002). Disponible en: https://nucleocdcht.files.wordpress.com/2013/05/6-sea-abril2002.pdf). Consultado el 24 de noviembre de 2018

Seibold, J. (2000). La calidad integral en educación. Reflexiones sobre un nuevo concepto de calidad educativa que integre valores y equidad educativa. Revista Iberoamericana de Educación, 23, 215-231 Recuperado en http://rieoei.org/rie23a07.htm

Silva, J (2009). Importancia de la Evaluación Institucional. Tomado el día 2 de diciembre de 2018. Disponible en: http://importanciaevaluacion.blogspot.com/

Senlle, A y Gutiérrez, N (2005). Calidad en los Servicios Educativos. Ediciones Díaz de Santos. España Paris.

UNESCO (2010): Informe de Seguimiento de la EPT en el Mundo 2010. Resumen 
Universidad Carlos III de Madrid (UC3M) (2017). Modelo CINDA. Tomado el día 23 de noviembre de 2018.2 Disponible en: http://portal.uc3m.es/portal/page/portal/actualidad_cientifica/noticias/universidades_cin $\underline{\text { da }}$

Vallone, B (2012). La evaluación institucional y la cultura de la calidad, una situación relativamente nueva. Revista Reflexión Académica. No XVIII. Universidad de Palermo. Argentina.

Vivas, M (1999). El modelo de la Calidad Total para las Instituciones Educativas. Tomado el día 24 de noviembre de 2018. Disponible en: http://www.saber.ula.ve/bitstream/handle/123456789/17019/art5_v8n2.pdf?sequence=1 $\underline{\text { \&isAllowed }=\mathrm{y}}$ 